\title{
Effects of nicotinamide and riboflavin on the biodesulfurization activity of dibenzothiophene by Rhodococcus erythropolis USTB-03
}

\author{
YAN Hai $^{1, *}$, SUN Xudong ${ }^{1}$, XU Qianqian ${ }^{1}$, MA Zhao ${ }^{1}$, XIAO Chengbin ${ }^{2}$, Ning Jun ${ }^{2}$ \\ 1. Department of Biological Science and Technology, School of Applied Science, University of Science and Technology Beijing, \\ Beijing 100083, China. E-mail: haiyan@sas.ustb.edu.cn \\ 2. Research Center for Eco-environmental Science, Chinese Academy of Sciences, Beijing 100085, China
}

Received 8 July 2007; revised 9 August 2007; accepted 20 August 2007

\begin{abstract}
Rhodococcus erythropolis USTB-03 is a promising bacterial strain for the biodesulfurization of dibenzothiophene (DBT) via a sulfurspecific pathway in which DBT is converted to 2-hydroxybiphenyl (2HBP) as an end product. The effects of nicotinamide and riboflavin on the sulfur specific activity (SA) of DBT biodesulfurization by $R$. erythropolis USTB-03 were investigated. Both nicotinamide and riboflavin were found to enhance the expression of SA, which was not previously reported. When $R$. erythropolis USTB-03 was grown on a medium containing nicotinamide of $10.0 \mathrm{mmol}$ or riboflavin of $50.0 \mu \mathrm{mol}$, SA was raised from 68.0 or so to more than $130 \mathrm{mmol}$ $2 \mathrm{HBP} /(\mathrm{kg}$ dry cells.h). When $R$. erythropolis USTB-03 was grown in the presence of both nicotinamide of 5.0 mmol and riboflavin of $25.0 \mu \mathrm{mol}, \mathrm{SA}$ was further increased to $159.0 \mathrm{mmol} 2 \mathrm{HBP} /(\mathrm{kg}$ dry cells $\cdot \mathrm{h})$. It is suggested that the biological synthesis of reduced form of flavin mononucleotide (FMNH2), an essential coenzyme for the activities of biodesulfurization enzyme Dsz C and A, might be enhanced by nicotinamide and riboflavin, which was responsible for the increased SA of $R$. erythropolis USTB-03.
\end{abstract}

Key words: biodesulfurization; dibenzothiophene; nicotinamide; Rhodococcus erythropolis USTB-03; riboflavin

\section{Introduction}

Combustion of sulfur-rich fossil fuels leads to the release of sulfur oxides that causes air pollution and acid rain, which has become a major environmental problem. The sulfur content ranges from $0.05 \%$ to $5.0 \%$ in crude oil and from $0.3 \%$ to $6.0 \%$ in coal (Shennan, 1996; Wang and Krawiec, 1994). On average, organic and inorganic sulfurs are present in equal amounts in coal, while the majority of sulfur compounds in liquid petroleum fuels are organic (Li et al., 1996). Although chemical-physical processes can be used to remove inorganic sulfur from crude fuels, organic sulfur in dibenzothiophene (DBT), a widely used model of organic sulfur compound, remains in petroleum and coal even after these processes (Gray et al., 1996). To overcome this problem, some promising bacterial strains are identified to remove sulfur from DBT through the sulfur-specific pathway, which is capable of obtaining nutritional sulfur from DBT and producing 2hydroxybiphenyl (2HBP) as the final product without causing oxidative loss of fuel carbon, which is the most efficient means for the desulfurization of DBT (Yan et al., 2000; Furuya et al., 2001; Kirimura et al., 2002; Li et al., 2005; Ishii et al., 2005; Yang and Marison, 2005; Gunam et al., 2006).

In the sulfur-specific pathway for the conversion of DBT

\footnotetext{
* Corresponding author. E-mail: haiyan@ sas.ustb.edu.cn.
}

to 2-HBP, four enzymes are involved in a four-step biochemical reaction: DBT monooxygenase (Dsz C) catalyses two consecutive monooxygenation reactions involving the conversion of DBT to DBT-sulfone $\left(\mathrm{DBTO}_{2}\right)$. Subsequently, a second flavomonooxygenase (Dsz A) is responsible for the conversion of $\mathrm{DBTO}_{2}$ to 2-2'-hydroxyphenyl benzene sulfinate (HPBS), and Dsz B, a desulfinase, catalyzes the final step involving the conversion of HPBS to $2 \mathrm{HBP}$ (Yan et al., 2000). Reduced form of flavin mononucleotide (FMNH2) is required for the activities of both Dsz C and $\mathrm{A}$, and a enzyme flavin reductase (Dsz D) is needed for the conversion of FMN to FMNH2 using NADH as the reducing agent (Lei and Tu, 1996; Matsubara et al., 2001; Gray and Mrachko, 2003). Overexpression of Dsz D enhances the overall rate of desulfurization, which can be further induced up to 100 -fold when flavin reductase is added to the biological reaction mixture, suggesting that the supply of reduced flavin is a rate-limiting step in the sulfur-specific pathway (Gray et al., 1996; Galan et al., 2000; Reichmuth et al., 2000; Ohshiro et al., 1995).

A significant stumbling block to the commercialization of biodesulfurization is the low specific activity (SA) for removing sulfur from organic compounds (Borgne and Quintero, 2003). If microorganisms are to be used as non-growing biocatalysts, they need to be grown in a separate process and SA of biodesulfurization is a crucial indicator for efficiency. Previously, high SA of $135.5 \mathrm{mmol}$ 
$2 \mathrm{HBP} /(\mathrm{kg}$ dry cells $\cdot \mathrm{h})$ and cell concentration of $37 \mathrm{~g}$ dry cells/L of Rhodococcus erythropolis KA2-5-1 were found in a fed-batch fermentation culture, when ethanol as the sole carbon and energy source was maintained at $1.0 \mathrm{~g} / \mathrm{L}$ under the control of a semiconductor gas sensor (Yan et al., 2000). The genes for biodesulfurization enzymes have been cloned, sequenced and engineered from a variety of microorganisms (Ishii et al., 2000; Borole et al., 2002; Nomura et al., 2005); however, little information was provided on how to increase SA of biodesulfurization through the metabolic promotion of FMNH2 and the production of reduced form of nicotinamide-adenine dinucleotide (NADH) .

In this study, the effects of nicotinamide and riboflavin on the biodesulfurization activity of DBT by $R$. erythropolis USTB-03 were investigated. It showed that addition of nicotinamide of $5.0 \mathrm{mmol}$ and riboflavin of $25.0 \mu \mathrm{mol}$ to the culture medium of $R$. erythropolis USTB-03 induced SA more than two-fold, from 69.0 to $159.0 \mathrm{mmol}$ $2 \mathrm{HBP} /(\mathrm{kg}$ dry cells $\cdot \mathrm{h})$. Further exploration of this process is warranted to obtain high SA of biodesulfurization for industrial application.

\section{Materials and methods}

\subsection{Strain and chemicals}

Rhodococcus erythropolis USTB-03 used was previously isolated from the contaminated soil at the petroleum refining company in Zhanjiang City, China (Wei et al., 2005). DBT and 2HBP were purchased from Wako Pure Chemical Industries, Japan. Nicotinamide and riboflavin were obtained from Sigma Chemical Co., USA.

\subsection{Medium and culture conditions}

The sulfur-free basal medium was the same as previously described (Izumi et al., 1994). Glucose of $15 \mathrm{~g} / \mathrm{L}$ and DBT of $50 \mathrm{mg} / \mathrm{L}$ were used as carbon and sulfur sources, respectively. Both medium and all experimental utensils were sterilized at $124^{\circ} \mathrm{C}$ for $20 \mathrm{~min}$. The solution of $1.0 \mathrm{~mol}$ nicotinamide or riboflavin filtered with a 0.22 $\mu \mathrm{m}$ embrane was added in the sterilized medium on a clean bench. The strain was grown in 100 ml-flasks containing $20 \mathrm{ml}$ inoculated liquid medium. The liquid culture was shaken at the rate of $200 \mathrm{r} / \mathrm{min}$ at $30^{\circ} \mathrm{C}$.

\subsection{Analytical methods}

Growth was measured turbid metrically at $680 \mathrm{~nm}$ $\left(\mathrm{OD}_{680}\right)$. To measure DBT and $2 \mathrm{HBP}$ concentrations in the medium, the culture broth was taken and directly extracted with $n$-hexane by shaking for $1.0 \mathrm{~min}$ on a vortex. After centrifugation at $12,000 \mathrm{r} / \mathrm{min}$ for $5 \mathrm{~min}$, the supernatant of the $n$-hexane extract was collected and injected into a HPLC (Shimadzu LC-10ATVP, Japan) with a UV Diode Array Detector at $240 \mathrm{~nm}$ using a SB-C18 $(4.6 \times 250 \mathrm{~mm}$, $5 \mu \mathrm{m})$ column from Agilent. The mobile phase was $95 \%$ $(V / V)$ methanol-water solution and the flow-rate was 1.0 $\mathrm{ml} / \mathrm{min}$. The calibration curves were established between the peak areas and the known concentrations of DBT and 2HBP in $n$-hexane, respectively, which were used to calculate the amount of DBT and 2HBP by peak area from unknown samples. The data presented here were the average of three measurements and their standard deviations were less than $10 \%$.

\subsection{Specific activity}

Samples from the culture medium were taken and centrifuged at 12,000 r/min for $5 \mathrm{~min}$. After discarding the supernatant, cells remained at the bottom of the tube were washed twice with $50 \mathrm{mmol}$ phosphate buffer solution (PB, $\mathrm{pH}$ 7.0). The washed cells mixed with DBT of $100 \mathrm{mg} / \mathrm{L}$ in PB of $10 \mathrm{ml}$ were directly used to measure SA. As a control, the cell suspension was mixed with $10 \mu \mathrm{l}$ of 12 mol hydrochloric acid to stop the reaction. The reaction was maintained at $30^{\circ} \mathrm{C}$ for $1 \mathrm{~h}$ with a shake rate of 200 $\mathrm{r} / \mathrm{min}$, and $10 \mu \mathrm{l}$ of $12 \mathrm{~mol}$ hydrochloric acid was added to stop the reaction. The measurement procedures of DBT and $2 \mathrm{HBP}$ were the same as those in the analytical methods described above. SA was expressed as the amount (mmol) of $2 \mathrm{HBP}$ produced per kg-dry cells per hour. The linear relationship between $\mathrm{OD}_{680}$ and dry weight concentrations of $R$. erythropolis USTB-03 was established as: dry weight concentration $(\mathrm{g} / \mathrm{L})=0.22 \mathrm{OD}_{680}-0.15$, which was used to calculate the dry weight of cells based on OD at $680 \mathrm{~nm}$.

\section{Results}

\subsection{Determination of DBT and 2HBP on HPLC}

Figure 1 is the HPLC chromatogram and scanning profiles of standard DBT and $2 \mathrm{HBP}$ of $15 \mathrm{mg} / \mathrm{L}$ in $n$ hexane, and the retention time of $2 \mathrm{HBP}$ and DBT were 3.1 and $5.3 \mathrm{~min}$, respectively (Fig.1a). The scanning profiles of DBT and $2 \mathrm{HBP}$ peaks in the wavelength range from 200 to $300 \mathrm{~nm}$ confirmed that the maximum absorbance of DBT and 2HBP were at 232 (Fig.1b) and $245 \mathrm{~nm}$ (Fig.1c), respectively. To measure DBT and 2HBP simultaneously,
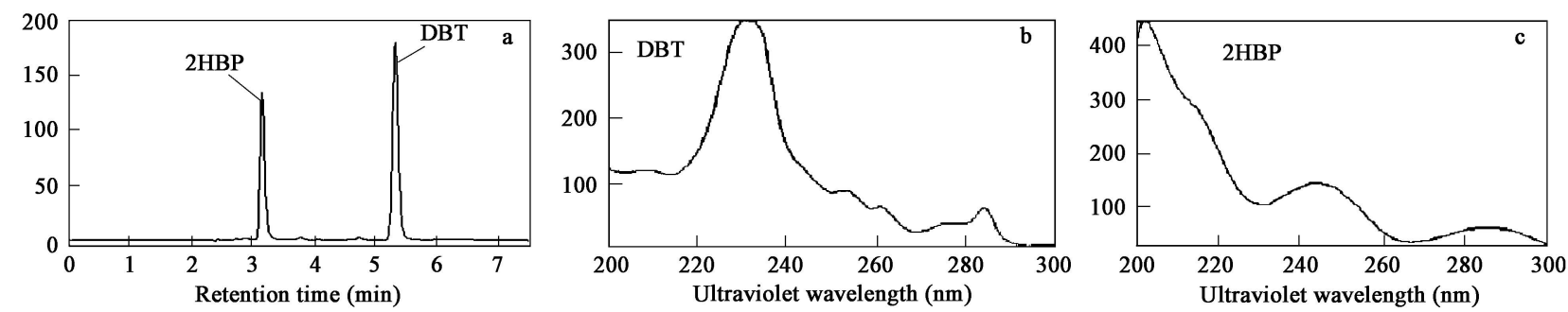

Fig. 1 HPLC chromatograms and scanning profiles of standard 2HBP and DBT of $15 \mathrm{mg} / \mathrm{L}$ in $n$-hexane. (a) HPLC chromatograms of $2 \mathrm{HBP}$ and DBT; (b) scanning profile of DBT in the ultraviolet wavelength from 200 to $300 \mathrm{~nm}$; (c) scanning profile of $2 \mathrm{HBP}$ in the ultraviolet wavelength from 200 to $300 \mathrm{~nm}$. 
the detection wavelength of $240 \mathrm{~nm}$ was used for the quantification of DBT and 2HBP. Using this method, the detection limits for $2 \mathrm{HBP}$ and DBT were found to be 0.4 and $0.3 \mathrm{mg} / \mathrm{L}$, respectively. The calibration curves in the range from 0 to $50 \mathrm{mg} / \mathrm{L}$ were established as: $\mathrm{DBT}(\mathrm{mg} / \mathrm{L})$ $=6.88$ peak area $\left(10^{6}\right)-0.47\left(R^{2}=0.994\right) ; 2 \mathrm{HBP}(\mathrm{mg} / \mathrm{L})=$ 1.31 peak area $\left(10^{5}\right)-0.61\left(R^{2}=0.991\right)$, which were used to calculate the concentrations of DBT and $2 \mathrm{HBP}$ based on their peak areas.

\subsection{Effect of nicotinamide on the growth and SA of $R$. erythropolis USTB-03}

Figure 2a shows the effect of nicotinamide on the bacterial growth rate. The growth of $R$. erythropolis USTB-03 at exponential phase of day 2 was gradually inhibited with the increase of nicotinamide from 0 to $20 \mathrm{mmol}$. However,
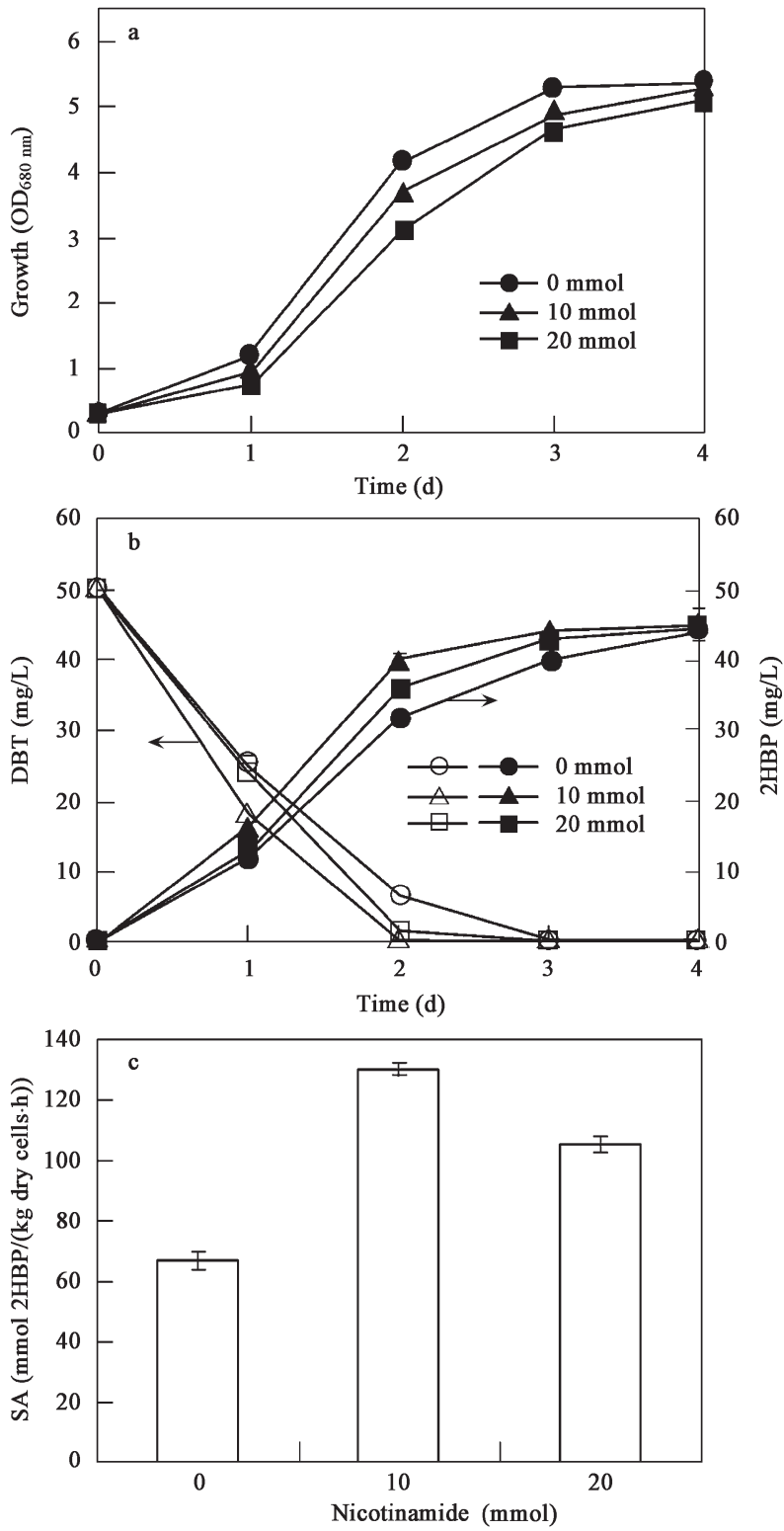

Fig. 2 Effect of nicotinamide on the growth and SA of R. erythropolis USTB-03. (a) effect of nicotinamide on the growth of $R$. erythropolis USTB-03; (b) removal of DBT (blank symbols) and the production of 2HBP (black symbols); (c) effect of nicotinamide on SA of $R$. erythropolis USTB-03. both the decline of DBT and the production of $2 \mathrm{HBP}$ were slightly induced in the presence of nicotinamide (Fig. $2 b)$. SA of $R$. erythropolis USTB-03 on day 4 was also improved when it was grown on nicotinamide (Fig.2c). Compared to that of $67.0 \mathrm{mmol} 2 \mathrm{HBP} /(\mathrm{kg}$ dry cells $\cdot \mathrm{h})$ in control, the maximum SA of $130.2 \mathrm{mmol} 2 \mathrm{HBP} /(\mathrm{kg}$ dry cells.h) was observed when USTB-03 was cultured in the presence of $10 \mathrm{mmol}$ nicotinamide (Fig.2c).

\subsection{Effect of riboflavin on the growth and SA of $R$. erythropolis USTB-03}

Similar to that of nicotinamide, Fig.3a demonstrates that the growth of $R$. erythropolis USTB-03 was slightly repressed by riboflavin in the range from 0 to $100 \mu \mathrm{mol}$, but the reduction of DBT and the production of $2 \mathrm{HBP}$ were enhanced (Fig.3b). The maximum SA of 135.0 compared with $68.6 \mathrm{mmol} 2 \mathrm{HBP} /(\mathrm{kg}$ dry cells $\cdot \mathrm{h})$ in control was achieved when $R$. erythropolis USTB-03 was grown on 50 $\mu$ mol riboflavin (Fig.3c).
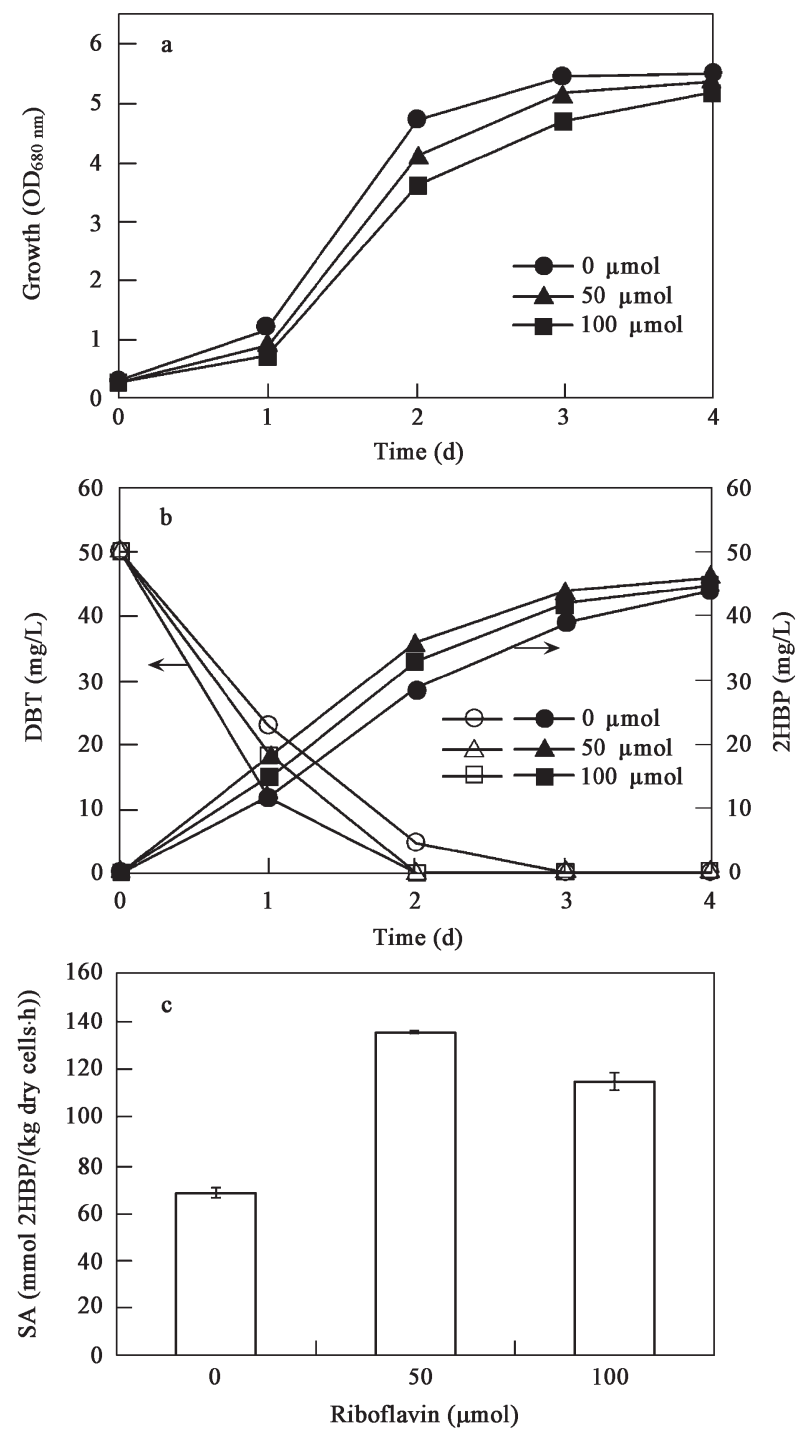

Fig. 3 Effect of riboflavin on the growth and SA of $R$. erythropolis USTB-03. (a) effect of riboflavin on the growth of $R$. erythropolis USTB03; (b) removal of DBT (blank symbols) and the production of $2 \mathrm{HBP}$ (black symbols); (c) effect of riboflavin on SA of R. erythropolis USTB03 . 


\subsection{Effect of nicotinamide and riboflavin on the growth and SA of R. erythropolis USTB-03}

Figure 4a shows that the growth of $R$. erythropolis USTB-03 at the exponential phase of day 2 was apparently inhibited by nicotinamide and riboflavin; however, the growth rate at stationary growth phases on day 4 was almost independent of nicotinamide and riboflavin. The reduction of DBT and the augment of $2 \mathrm{HBP}$ were also promoted when nicotinamide and riboflavin were simultaneously added to the culture medium (Fig.4b). The maximum SA of $159.0 \mathrm{mmol} 2 \mathrm{HBP} /(\mathrm{kg}$ dry cells $\cdot \mathrm{h})$ was achieved when $R$. erythropolis USTB-03 was cultured with initial nicotinamide of $5 \mathrm{mmol}$ and riboflavin of $25 \mu \mathrm{mol}$ (Fig.4c).
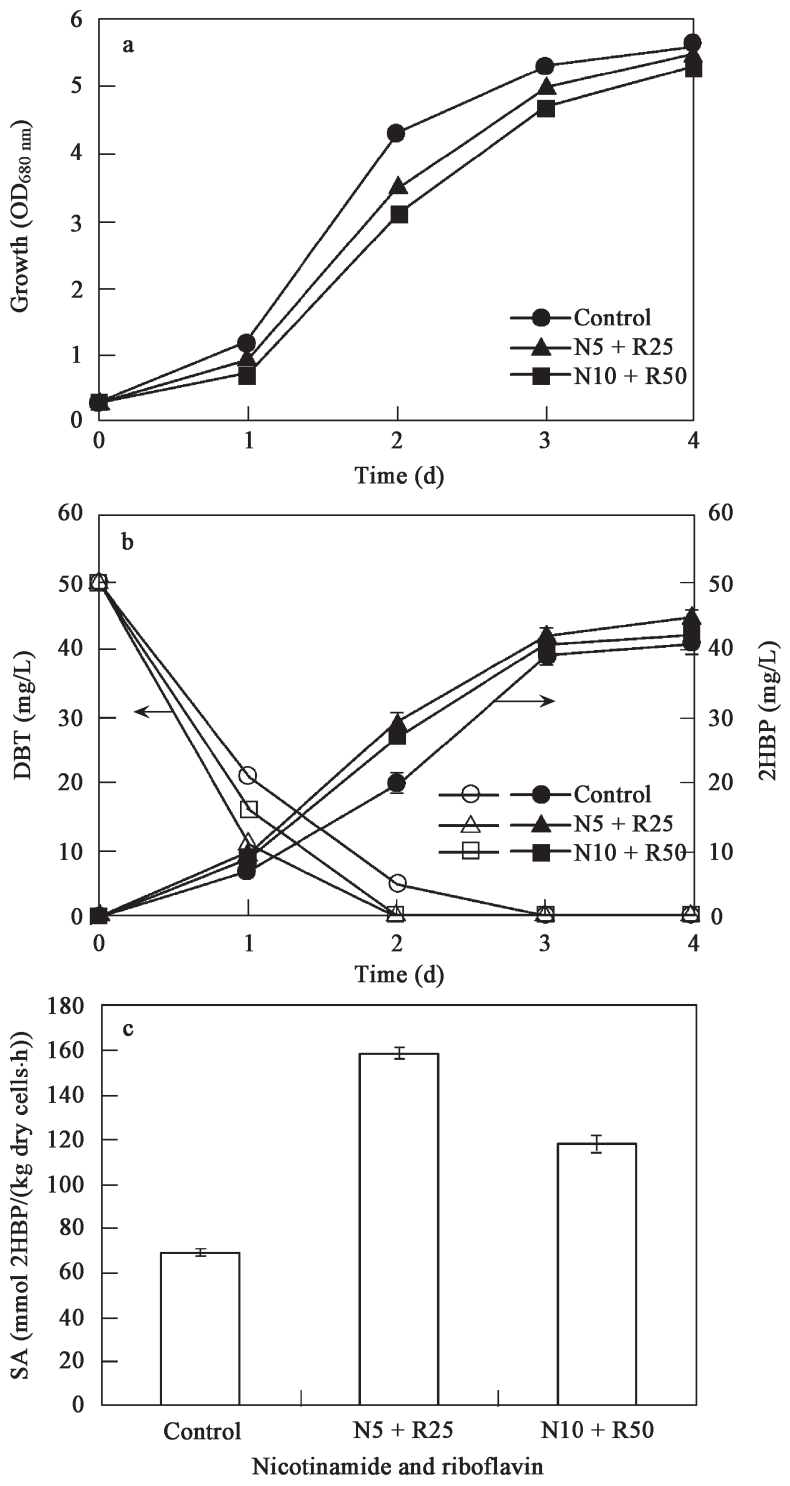

Fig. 4 Effects of nicotinamide and riboflavin on the growth and SA of $R$. erythropolis USTB-03. (a) effects of nicotinamide and riboflavin on the growth of $R$. erythropolis USTB-03; (b) removal of DBT (blank symbols) and the production of 2HBP (black symbols); (c) effects of nicotinamide and riboflavin on SA of $R$. erythropolis USTB-03. Control: nicotinamide $0 \mathrm{mmol}$ and riboflavin $0 \mu \mathrm{mol}$; $\mathrm{N} 5+\mathrm{R} 25$ : nicotinamide 5 mmol and riboflavin $25 \mu \mathrm{mol}$; N10+R50: nicotinamide $10 \mathrm{mmol}$ and riboflavin $50 \mu \mathrm{mol}$.

\section{Discussion}

\subsection{Determination of DBT and 2HBP on HPLC}

Quantitative measurement of DBT and $2 \mathrm{HBP}$ were previously achieved using GC. In this study, we report a method of measurement using HPLC (Fig.1). The maximum absorbance of DBT and 2HBP were found to be at the wavelengths of 232 and $245 \mathrm{~nm}$, respectively. The wavelength of $240 \mathrm{~nm}$ was chosen to determine both DBT and 2HBP simultaneously, which ensured the veracious detection of DBT and 2HBP throughout the experiment.

\subsection{Effects of nicotinamide and riboflavin on the growth of $\boldsymbol{R}$. erythropolis USTB-03}

Results showed that the growth $\left(\mathrm{OD}_{680}\right)$ of R. erythropolis USTB-03 at exponential phases of day 2 were gradually repressed with the increase in the initial concentrations of nicotinamide and riboflavin; however, the final $\mathrm{OD}_{680}$ at stationary phases of day 4 remained almost independent of nicotinamide or riboflavin (Figs.2a, 3a, and 4a). Although the exponential growth of $R$. erythropolis USTB-03 was apparently delayed, the biomass obtained at stationary phase of day 4 was hardly affected (Figs.2a, 3a, and 4a), which ensured to obtain the the enough biomass of $\mathrm{R}$. erythropolis USTB-03 for the in the biodesulfurization of DBT.

\subsection{Effects of nicotinamide and riboflavin on SA of $R$. erythropolis USTB-03}

Both the reduction of DBT and the production of $2 \mathrm{HBP}$ were enhanced (Figs.2b, 3b and 4b), although the growth of $R$. erythropolis USTB-03 was slightly inhibited by nicotinamide and riboflavin (Figs.2a, 3a and 4a). Addition of nicotinamide or riboflavin was found to obviously promote SA of R. erythropolis USTB-03 (Figs.2c and 3c). The current maximum SA of $159.0 \mathrm{mmol} 2 \mathrm{HBP} /(\mathrm{kg}$ dry cells $\cdot \mathrm{h})$, in comparison with $135.0 \mathrm{mmol} 2 \mathrm{HBP} /(\mathrm{kg}$ dry cells.h) in our previous report (Yan et al., 2000), was et al., 2000), was achieved when $R$. erythropolis USTB-03 was grown on both nicotinamide of $5 \mathrm{mmol}$ and riboflavin of $25 \mu \mathrm{mol}$ (Fig.4c). In addition to the enhancement of SA by genetic engineering was widely studied (Ishii et al., 2000; Borole et al., 2002; Nomura et al., 2005), it could also be achieved by the metabolic supplementation using nicotinamide and riboflavin in the culture medium.

In the sulfur-specific pathway, the first step involving the conversion of DBT to DBT sulfoxide is the rate-limiting step (Yan et al., 2000). Both enzyme Dsz C and D are involved in catalyzing this step of biochemical reaction. Because Dsz D, responsible for the conversion of FMN to FMNH2 using NADH as the reducing agent, is required for the activity of Dsz C. Both NADH and FMNH2 play vital roles in the biosulfurization of DBT (Lei and Tu, 1996; Matsubara et al., 2001; Gray and Mrachko, 2003; Furuya et al., 2005). As nicotinamide and riboflavin are the precursors of NAD and FMN, it may easily be converted to NADH and FMNH2, respectively. So both the removal of DBT and the production of $2 \mathrm{HBP}$ were 
enhanced during the growths of $R$. erythropolis USTB-03 when nicotinamide and riboflavin were added to the culture medium (Figs.2b, 3b and 4b). SA of R. erythropolis USTB03 grown on nicotinamide or riboflavin was increased from 68 or so to more than $100 \mathrm{mmol} 2 \mathrm{HBP} /(\mathrm{kg}$ dry cells.h) (Figs.2c and 3c), and the maximum SA of 159.0 mmol $2 \mathrm{HBP} /(\mathrm{kg}$ dry cells·h) was further achieved when $R$. erythropolis USTB-03 was cultured in the presence of both $10 \mathrm{mmol}$ nicotinamide and $25 \mu \mathrm{mol}$ riboflavin (Fig.4c).

For the production of NADH from nicotinamide, Evans et al. (2002) found that nicotinamide of $50 \mu \mathrm{mol}$ was the dominant precursor and NAD levels in the cell rose well above normal levels. Reduction of NAD to NADH by $S$. cerevisiae during assimilation of biomass from glucose was reported by Bakker et al. (2001). Because the conversion of ethanol to acetaldehyde catalyzed by alcohol dehydrogenase might promote the production of NADH from NAD, the increase in SA of $R$. erythropolis KA2-51 was indicated (Yan et al., 2000). For the formation of FMNH2, Bauer et al. (2003) reported that the conversion of riboflavin to FMN was catalyzed by riboflavin kinase, which was further converted to FMNH2 using NADH as the reducing agent (Gray et al., 1996; Lei and Tu, 1996; Matsubara et al., 2001; Furuya et al., 2005; Bauer et al., 2003). Here we suggest that the production of FMNH2 as a key coenzyme for the activities of Dsz C and A can be promoted by NADH and FMN that can be enhanced by NAD and riboflavin, and NAD is easily produced by nicotinamide, which is responsible for the increased SA of $R$. erythropolis USTB-03.

Although microbial biodesulfurization from unprocessed fuel may be useful for environmental protection, the low efficiency may block commercialization of biodesulfurization process. Here we report that the improvement of a crucial indicator SA of $R$. erythropolis USTB-03 can be attained by adding suitable amounts of nicotinamide and riboflavin in culture medium. Using little amount of riboflavin and nicotinamide as precursors of FMNH2 and NADH, SA of $R$. erythropolis USTB-03 is much increased, which will be very useful in the cultivation of $R$. erythropolis USTB-03 with high SA for the effective biodesulfurization of DBT in practical application.

\section{Conclusions}

The effects of nicotinamide and riboflavin on the biodesulfurization activity of DBT by $R$. erythropolis USTB-03 were investigated in this study. Both the removal of DBT and the production of $2 \mathrm{HBP}$ by $R$. erythropolis USTB-03 were apparently enhanced by the addition of suitable concentrations of nicotinamide and riboflavin into the culture medium. SA of $68.0 \mathrm{mmol} 2 \mathrm{HBP} /(\mathrm{kg}$ dry cells $\cdot \mathrm{h})$ increased to 130.2 or $135.0 \mathrm{mmol} 2 \mathrm{HBP} /(\mathrm{kg}$ dry cells $\cdot \mathrm{h})$ at initial nicotinamide concentration of $10 \mathrm{mmol}$ or riboflavin of $50 \mu \mathrm{mol}$, respectively. The maximal SA of $159.0 \mathrm{mmol} 2 \mathrm{HBP} /(\mathrm{kg}$ dry cells $\cdot \mathrm{h})$ was obtained when $R$. erythropolis USTB-03 was grown on both nicotinamide of $5 \mathrm{mmol}$ and riboflavin of $25 \mu \mathrm{mol}$. We hypothesize that addition of nicotinamide and riboflavin may promote the biological synthesis of FMNH2, an essential coenzyme for Dsz $\mathrm{C}$ and $\mathrm{A}$, which is very important in the increase of SA of $R$. erythropolis USTB-03 for the effective biodesulfurization of DBT in practical application.

\section{Acknowledgements}

This work was supported by the National Natural Science Foundation of China (No. 20777008) and the Innovation Foundation in University of Science and Technology Beijing. We are indebted to Dr. Qing Song for the revision of this paper.

\section{References}

Bakker B M, Overkamp K M, Maris A J A, Kotter P, Luttik M A H, Dijken J P, Pronk J T, 2001. Stoichiometry and compartmentation of NADH metabolism in Saccharomyces cerevisiae. FEMS Microbiol Rev, 25: 15-37.

Bauer S, Kemter K, Bacher A, Huber R, Fischer M, Steinbacher S, 2003. Crystal structure of schizosaccharomyces pombe riboflavin kinse reveals a novel ATP and riboflavin-binding fold. J Mol Biol, 326: 1463-1473.

Borgne S L, Quintero R, 2003. Biotechnological processes for the refining of petroleum. Fuel Processing Technol, 81: 155169.

Borole A P, Kaufman E N, Grossman M J, Minak-Bernero V, Bare R, Lee M K, 2002. Comparison of the emulsion characteristics of Rhodococcus erythropolis and Ecsherichia coli SOXC-5 cells expressing biodesulfurization genes. Biotechnol Progr, 18: 88-93.

Evans J, Wang T C, Heyes M P, Markey S P, 2002. LC/MS analysis of NAD biosynthesis using stable isotope pyridine precursors. Anal Biochem, 306: 197-203.

Furuya T, Kirimura K, Kino K, Usami S, 2001. Thermophilic biodesulfurization of dibenzothiophene and its derivatives by Mycobacterium phlei WU-F1. FEMS Microbiol Lett, 204: 129-133.

Furuya T, Takahashi S, Iwasaki Y, Ishii Y, Kino K, Kirimura K, 2005. Gene cloning and characterization of Mycobacterium phlei flavin reductase involved in dibenzothiophene desulfurization. $J$ Biosci and Bioeng, 99: 577-585.

Galan B, Diaz E, Garcia J L, 2000. Enhancing desulfurization by engineering a flavin-reductase-encoding gene cassotte in recombinant biocatalysts. Environ Microbiol, 2: 687-694.

Gray K A, Mrachko G T, Squires C H, 2003. Biodesulfurization of fossil fuels. Curr Opin Microbiol, 6: 229-235.

Gray K A, Pogrebinsky O S, Mrachko G T, Lei X, Monticello D J, Squires C H, 1996. Molecular mechanisms of biocatalytic desulfurization of fossil fuels. Nat Biotechnol, 14: 17051709.

Gunam I B W, Yaku Y, Hirana M, Yamamura K, Tomita F, Sone T, Asano K, 2006. Biodesulfurization of alkylated forms of dibenzothiophene and benzothiophene by Sphingomonas subarctica T7b. J Biosci and Bioeng, 101: 322-327.

Ishii Y, Konishi J, Okada H, Hirasawa K, Onaka T, Suzuki M, 2000. Operon structure and functional analysis of the genes encoding thermophilic desulfurizing enzymes of Paenibacillus sp. A11-2. Biochem and Biophy Res Co, 270: 81-88.

Ishii Y, Kozaki S, Furuya T, Kino K, Kirimura K, 2005. Thermophilic biodesulfurization of various heterocyclic sulfur compounds and crude straight-run light gas oil fraction by a newly isolated strain Mycobacterium phlei WU-0103. Curr 
Microbiol, 50: 63-70.

Izumi Y, Ohshiro T, Ogino H, Hine Y, Shimao M, 1994. Selective desulfurization of dibenzothiophene by Rhodococcus erythropolis D-1. Appl Environ Microbiol, 60: 223-226.

Kirimura K, Furuya T, Sato R, Ishii Y, Kino K, Usami S, 2002. Biodesulfurization of naphthothiophene and benzothiophene through selective cleavage of carbon-sufur bonds by Rhodococcus sp. strain WU-K2R. Appl and Environ Microbiol, 68: 3867-3872.

Lei B F, Tu S C, 1996. Gene overexpression, purification, and identification of a desulfurization enzyme from Rhodococcus sp. strain IGST8 as a sulfide/sulfoxide monooxygenase. J Bacteriol, 178: 5699-5705.

Li M Z, Squires C H, Monticello D J, Childs D J, 1996. Genetic analysis of the dsz promoter and associated regulatory regions of Rhodococcus erythropolis IGTS8. J Bacteriol, 178: 6409-6418.

Li W, Zhang Y, Wang M D, Shi Y, 2005. Biodesulfurization of dibenzothiophene and other organic sulfur compounds by a newly isolated microbacterium strain ZD-M2. FEMS Microbiol Lett, 247: 45-50.

Matsubara T, Ohshiro T, Nishina Y, Izumi Y, 2001. Purification, characterization, and overexpression of flavin reductase involved 298 in dibenzothiophene desulfurization by Rhodococcus erythropolis D-1. Appl Environ Microbiol, 67: 1179-1184.

Nomura N, Takada M, Okada H, Shinohara Y, Nakajima-Kambe T, Nakahara T, Uchiyama H, 2005. Identification and func- tional analysis of genes required for desulfurization of alkyl dibenzothiophenes of Mycobacterium sp. G3. J Biosci and Bioeng, 100: 398-402.

Ohshiro T, Kanbayashi Y, Hine Y, Izumi Y, 1995. Involvement of flavin coenzyme in dibenzothiophene degrading enzyme system from Rhodococcus erythropolis D-1. Biosci Biotechnol Bioch, 59: 1349-1351.

Reichmuth D S, Hittle J L, Blanch H W, Keasling J D, 2000. Biodesulfurization of dibenzothiophene in Escherichia coli is enhanced by expression of a Vibrio harveyi oxidoreductase gene. Biotechnol and Bioeng, 67: 72-79.

Shennan J L, 1996. Microbial attack on suphur-containing hydrocarbons: implications for the biodesulphurization of oils and coals. J Chem Technol Biotechnol, 67: 109-123.

Wang P, Krawiec S, 1994. Desulfurization of dibenzothiophene to 2 hydroxybiphenyl by some newly isolated bacterial strains. Arch Microbiol, 161: 266-271.

Wei Y X, Yan H, Xiao B Q, Li Y X, Lv L, 2005. Biodesulfurization of 4,6-dimethyl dibenzothiophene by Rhodococcus erythropolis USTB03. Sci and Technol Rev, 23: 10-13.

Yan H, Kishimoto M, Omasa T, Katakura Y, Suga K I, Okumura $\mathrm{K}$, Yoshikawa Y, 2000. Increase in desulfurization activity of Rhodococcus erythropolis KA2-5-1 using ethanol feeding. J Biosci and Bioeng, 89: 361-366.

Yang JZ, Marison I W, 2005. Two-stage process design for the biodesulfurization of a model diesel by a newly isolated Rhodococcus globerulus DAQ3. Biochem Eng J, 27: 77-82. 